\title{
ON G-REGULAR LOCAL RINGS
}

\author{
RYO TAKAHASHI
}

\begin{abstract}
In this paper, we define a G-regular local ring as a commutative, noetherian, local ring over which all totally reflexive modules are free. We study G-regular local rings, and observe that they behave similarly to regular local rings. We extend Eisenbud's matrix factorization theorem and Knörrer's periodicity theorem to G-regular local rings.
\end{abstract}

\section{INTRODUCTION}

In the 1960s, Auslander [1] introduced a homological invariant for finitely generated modules over a noetherian ring which is called Gorenstein dimension, or G-dimension for short. After that, he developed the theory of G-dimension with Bridger [2]. G-dimension has been studied deeply from various points of view; details can be found in [2] and [8].

Modules of G-dimension zero are called totally reflexive modules. Any finitely generated projective module is totally reflexive. Over a Gorenstein local ring, the totally reflexive modules are precisely the maximal Cohen-Macaulay modules. Therefore, every singular Gorenstein local ring has a nonfree totally reflexive module.

In the present paper, we will call a commutative noetherian local ring $G$-regular if every totally reflexive module over the ring is free. Regular local rings are trivial examples of G-regular local rings. Avramov and Martsinkovsky [5, Examples 3.5] proved that any Golod local ring that is not a hypersurface (e.g. a Cohen-Macaulay non-Gorenstein local ring with minimal multiplicity [3, Example 5.2.8]) is G-regular. Yoshino [22, Theorem 3.1] gives some sufficient conditions for an artinian local ring of Loewy length three to be G-regular. Takahashi and Watanabe [19, Theorem 1.1] showed that there exist two-dimensional, non-G-regular, non-Gorenstein normal domains. A recent result due to Christensen, Piepmeyer, Striuli and Takahashi [9, Theorem B] says that every non-Gorenstein local ring over which there exist only finitely many isomorphism classes of indecomposable totally reflexive modules is a G-regular ring. The same result in special cases and similar results were earlier shown in [13]-[18].

In this paper we find that G-regular local rings behave similarly to regular local rings. We give two theorems, stated below, as the main results of this paper. The first is a generalization of Eisenbud's matrix factorization theorem [10, Section 6] (cf. [21, Theorem (7.4)]), and the second is a generalization of Knörrer's periodicity theorem [11, Theorem 3.1].

Let $S$ be a G-regular local ring, $f \in S$ an $S$-regular element, and $R=S /(f)$ the residue ring. We denote by $\mathcal{M}_{S}(f)$ the quotient category of the category of matrix factorizations of $f$ over $S$ by the matrix factorization $(1, f)$, by $\underline{\mathcal{M}}_{S}(f)$ the quotient category of $\mathcal{M}_{S}^{0}(f)$ by $(f, 1)$, by $\mathcal{G}(R)$ the category of totally reflexive $R$-modules, and by $\underline{\mathcal{G}}(R)$ the stable category of $\mathcal{G}(R)$.

Theorem A (matrix factorization). There are equivalences of categories:

$$
\begin{aligned}
& \mathcal{M}_{S}(f) \simeq \mathcal{G}(R), \\
& \underline{\mathcal{M}}_{S}(f) \simeq \underline{\mathcal{G}}(R) .
\end{aligned}
$$

Theorem B (Knörrer's periodicity). Let $B=S[[x, y]] /(f+x y)$.

(1) There is a fully faithful functor $\Delta: \underline{\mathcal{G}}(R) \rightarrow \underline{\mathcal{G}}(B)$.

(2) Suppose that $\frac{1}{2}, \sqrt{-1} \in S$ and that $\stackrel{R}{R}$ is henselian. Then the functor $\Delta$ is an equivalence.

2000 Mathematics Subject Classification. 13H10, 13C13, $13 \mathrm{D} 05$.

Key words and phrases. G-regular local ring, totally reflexive module, G-dimension, matrix factorization, Knörrer's periodicity. 


\section{BASIC DEFINITIONS}

In this paper we use commutative noetherian rings and their categories of finitely generated modules. In this section let $R$ be a local ring with maximal ideal $\mathfrak{m}$ and residue field $k$, and let $\bmod R$ denote the category of finitely generated $R$-modules. A subcategory always means a full subcategory closed under isomorphism.

Definition 1.1. (1) Let $(-)^{*}$ denote the $R$-dual functor $\operatorname{Hom}_{R}(-, R)$. An $R$-module $M$ is called totally reflexive if

(i) the natural homomorphism $M \rightarrow M^{* *}$ is an isomorphism, and

(ii) $\operatorname{Ext}_{R}^{i}(M, R)=\operatorname{Ext}_{R}^{i}\left(M^{*}, R\right)=0$ for any $i>0$.

(2) Let $M$ be a nonzero $R$-module. If there exists an exact sequence

$$
0 \rightarrow X_{n} \rightarrow X_{n-1} \rightarrow \cdots \rightarrow X_{1} \rightarrow X_{0} \rightarrow M \rightarrow 0
$$

of $R$-modules such that each $X_{i}$ is totally reflexive, then we say that $M$ has $G$-dimension at most $n$. If such an integer $n$ does not exist, then we say that $M$ has infinite $G$-dimension, and write $\operatorname{Gdim}_{R} M=\infty$. If $M$ has G-dimension at most $n$ but does not have G-dimension at most $n-1$, then we say that $M$ has $G$-dimension $n$, and write $\operatorname{Gdim}_{R} M=n$. We set $\operatorname{Gdim}_{R} 0=-\infty$.

Remark 1.2. An $R$-module $M$ is totally reflexive if and only if $\operatorname{Gdim}_{R} M \leq 0$.

Definition 1.3. A subcategory $\mathcal{X}$ of $\bmod R$ is called resolving if it satisfies the following four conditions.

(1) $\mathcal{X}$ contains $R$.

(2) $\mathcal{X}$ is closed under direct summands: if $M$ is an $R$-module in $\mathcal{X}$ and $N \oplus P \cong M$, then $N$ is also in $\mathcal{X}$.

(3) $\mathcal{X}$ is closed under extensions: for an exact sequence $0 \rightarrow L \rightarrow M \rightarrow N \rightarrow 0$ of $R$-modules, if $L$ and $N$ are in $\mathcal{X}$, then $M$ is also in $\mathcal{X}$.

(4) $\mathcal{X}$ is closed under kernels of epimorphisms: for an exact sequence $0 \rightarrow L \rightarrow M \rightarrow N \rightarrow 0$ of $R$-modules, if $M$ and $N$ are in $\mathcal{X}$, then $L$ is also in $\mathcal{X}$.

A resolving subcategory is a subcategory such that any two "minimal" resolutions of a module by modules in it have the same length; see [2, (3.12)].

Here we introduce three subcategories of $\bmod R$.

Notation 1.4. We denote by $\mathcal{F}(R)$ the subcategory of $\bmod R$ consisting of all free $R$-modules, by $\mathcal{G}(R)$ the subcategory of $\bmod R$ consisting of all totally reflexive $R$-modules, and by $\mathcal{C}(R)$ the subcategory of $\bmod R$ consisting of all $R$-modules $M$ satisfying the inequality $\operatorname{depth}_{R} M \geq \operatorname{depth} R$.

Let $M$ be an $R$-module. Take a minimal free resolution

$$
F_{\bullet}=\left(\cdots \stackrel{d_{3}}{\rightarrow} F_{2} \stackrel{d_{2}}{\rightarrow} F_{1} \stackrel{d_{1}}{\rightarrow} F_{0} \rightarrow 0\right)
$$

of $M$. For a nonnegative integer $n$, we set $\Omega_{R}^{n} M=\operatorname{Im} d_{n}$ and call it the $n$th syzygy of $M$. Note that the $n$th syzygy of a given $R$-module is uniquely determined up to isomorphism.

We will often use the following lemma. The assertion (1) is proved in [8, Theorem (1.4.9)], (2) in [7, Theorem 1.3.3] and [8, Theorem (1.4.8)], (3) in [8, Corollary (1.4.6) and Theorem (2.2.8)], and (4) in [8, Corollary (1.2.9)].

Lemma 1.5. (1) The following are equivalent:

(i) $R$ is Gorenstein;

(ii) $\operatorname{Gdim}_{R} M<\infty$ for all $R$-modules $M$;

(iii) $\operatorname{Gdim}_{R} k<\infty$.

(2) Let $M$ be an $R$-module.

(i) If $\operatorname{pd}_{R} M<\infty$, then $\operatorname{pd}_{R} M=\operatorname{depth} R-\operatorname{depth}_{R} M$.

(ii) If $\operatorname{Gdim}_{R} M<\infty$, then $\operatorname{Gdim}_{R} M=\operatorname{depth} R-\operatorname{depth}_{R} M$.

(3) Let $M$ be an $R$-module and $\boldsymbol{x}=x_{1}, \ldots, x_{n}$ a sequence of elements of $R$.

(i) If $\boldsymbol{x}$ is an $R$ - and $M$-sequence, then $\operatorname{Gdim}_{R /(\boldsymbol{x})} M / \boldsymbol{x} M=\operatorname{Gdim}_{R} M$.

(ii) If $\boldsymbol{x}$ is an $R$-sequence in $\operatorname{Ann}_{R} M$, then $\operatorname{Gdim}_{R /(\boldsymbol{x})} M=\operatorname{Gdim}_{R} M-n$.

(4) For an $R$-module $M$ and a nonnegative integer $n, \operatorname{Gdim}_{R} \Omega^{n} M=\sup \left\{\operatorname{Gdim}_{R} M-n, 0\right\}$.

Remark 1.6. The following are basic properties of the subcategories $\mathcal{F}(R), \mathcal{G}(R)$ and $\mathcal{C}(R)$. 
(1) All of $\mathcal{F}(R), \mathcal{G}(R)$ and $\mathcal{C}(R)$ are resolving subcategories of $\bmod R$.

(2) If $R$ is Cohen-Macaulay, then $\mathcal{C}(R)$ consists of all maximal Cohen-Macaulay $R$-modules.

(3) $\mathcal{C}(R)$ contains $\mathcal{G}(R)$, and $\mathcal{G}(R)$ contains $\mathcal{F}(R)$

(4) $R$ is Gorenstein if and only if $\mathcal{C}(R)$ coincides with $\mathcal{G}(R)$.

(5) $R$ is regular if and only if $\mathcal{C}(R)$ coincides with $\mathcal{F}(R)$.

The fact that $\mathcal{G}(R)$ is resolving is shown in [2, (3.11)] and [5, Lemma 2.3]. The first assertion in (3) follows from Lemma 1.5(2). As to (4), if $R$ is Gorenstein, then $\mathcal{C}(R)$ consists of all totally reflexive $R$-modules by Lemma 1.5(1) and (2). Conversely, suppose that $\mathcal{C}(R)$ coincides with $\mathcal{G}(R)$. Putting $t=\operatorname{depth} R$, we have depth $\Omega_{R}^{t} k=t$. Hence $\Omega_{R}^{t} k$ is in $\mathcal{C}(R)=\mathcal{G}(R)$. This implies that the $R$-module $k$ has G-dimension (at most) $t$, and thus $R$ is Gorenstein by Lemma 1.5(1). The assertion (5) is shown similarly to (4).

Definition 1.7. We say that a local ring $R$ is $G$-regular if $\mathcal{G}(R)$ coincides with $\mathcal{F}(R)$.

Proposition 1.8. (1) A local ring is regular if and only if it is G-regular and Gorenstein.

(2) A local ring $R$ is $G$-regular if and only if $\operatorname{Gdim}_{R} M=\operatorname{pd}_{R} M$ for any $R$-module $M$.

(3) A normal local ring $R$ is $G$-regular if and only if $\operatorname{Gdim}_{R} R / I=\operatorname{pd}_{R} R / I$ for any ideal $I$ of $R$.

Proof. (1) The assertion immediately follows from Remark 1.6(4) and (5).

(2) This can easily be shown using the definition.

(3) Let $M$ be a totally reflexive $R$-module. Then $M$ is reflexive, so $M$ is torsionfree. Hence there exists an exact sequence $0 \rightarrow R^{n} \rightarrow M \rightarrow I \rightarrow 0$ such that $I$ is an ideal of $R$; see $[6$, Theorem 6 in Chapter VII $\S 4]$. We obtain an exact sequence

$$
0 \rightarrow R^{n} \rightarrow M \rightarrow R \rightarrow R / I \rightarrow 0 .
$$

It follows by definition that the $R$-module $R / I$ has G-dimension at most 2 . If the equality $\operatorname{Gdim}_{R} R / I=$ $\operatorname{pd}_{R} R / I$ holds, then the $R$-module $R / I$ has finite projective dimension, and so does $M$. Thus $M$ is free by Lemma $1.5(2)$.

\section{MATRIX FACTORIZATIONS}

In this section, we generalize Eisenbud's matrix factorization theorem [10]. Throughout this section, let $S$ be a G-regular local ring with maximal ideal $\mathfrak{n}, f \in \mathfrak{n}$ an $S$-regular element, and $R=S /(f)$ the residue ring. First of all, let us make the definition of a matrix factorization.

Definition 2.1. For a nonnegative integer $n$, we call a pair $(\phi, \psi)$ of $n \times n$ matrices over $S$ a $\operatorname{matrix}$ factorization of $f$ (over $S$ ) if $\phi \psi=\psi \phi=f I_{n}$, where $I_{n}$ is the identity matrix. When $n=0$, both $\phi$ and $\psi$ can be considered as the $0 \times 0$ matrix over $S$ which we denote by $\zeta$, and we call the matrix factorization $(\zeta, \zeta)$ the zero matrix factorization of $f$.

Remark 2.2. If $(\phi, \psi)$ is a matrix factorization of $f$, then so are $(\psi, \phi),\left({ }^{t} \phi,{ }^{t} \psi\right)$ and $\left({ }^{t} \psi,{ }^{t} \phi\right)$, where ${ }^{t}(-)$ denotes the transpose.

In what follows, we will often identify an $m \times n$ matrix over $S$ with a homomorphism $S^{n} \rightarrow S^{m}$ of free $S$-modules. Thus the matrix $\zeta$ gives the identity map of the free $S$-module $S^{0}=0$ of rank zero.

A matrix factorization corresponds to an $R$-module which has projective dimension at most one as an $S$-module, as we see next.

Proposition 2.3. (1) Let $(\phi, \psi)$ be a matrix factorization of $f$. Then $M:=$ Coker $\phi$ is an $R$-module and there is an exact sequence $0 \rightarrow S^{n} \stackrel{\phi}{\rightarrow} S^{n} \rightarrow M \rightarrow 0$ in $\bmod S$.

(2) Let $M$ be an $R$-module and suppose that there is an exact sequence $0 \rightarrow S^{n} \stackrel{\phi}{\rightarrow} S^{m} \rightarrow M \rightarrow 0$ in $\bmod S$. Then one has $m=n$, and there is a matrix $\psi$ such that $(\phi, \psi)$ is a matrix factorization of $f$.

Proof. (1) By using the equalities $\phi \psi=\psi \phi=f I_{n}$, we easily see that $f M=0$ and that the endomorphism $\phi$ is injective over $S$.

(2) The equality $f M=0$ implies $M_{f}=0$. Hence we see that $m=n$. For each $x \in S^{n}$ we have $f x \in f S^{n} \subseteq \operatorname{Im} \phi$, and the injectivity of $\phi$ shows that there uniquely exists $y \in S^{n}$ such that $f x=\phi(y)$. Defining an endomorphism $\psi: S^{n} \rightarrow S^{n}$ by $\psi(x)=y$, we have $\phi \psi=f \cdot \operatorname{id}_{S^{n}}$. We get $\phi\left(\psi \phi-f \cdot \operatorname{id}_{S^{n}}\right)=0$, and $\psi \phi=f \cdot \operatorname{id}_{S^{n}}$ by the injectivity of $\phi$ again. It follows that $(\phi, \psi)$ is a matrix factorization of $f$.

Each matrix factorization of $f$ gives rise to a totally reflexive $R$-module. 
Proposition 2.4. Let $(\phi, \psi)$ be a matrix factorization of $f$, and let $n$ be the (common) size of the matrices $\phi$ and $\psi$. Then the sequence

$$
\cdots \stackrel{\phi}{\rightarrow} R^{n} \stackrel{\psi}{\rightarrow} R^{n} \stackrel{\phi}{\rightarrow} R^{n} \stackrel{\psi}{\rightarrow} \cdots
$$

is an exact sequence of $R$-modules whose $R$-dual is also exact. Hence $\operatorname{Coker}\left(S^{n} \stackrel{\phi}{\rightarrow} S^{n}\right) \cong \operatorname{Coker}\left(R^{n} \stackrel{\phi}{\rightarrow}\right.$ $\left.R^{n}\right)$ is a totally reflexive $R$-module.

Proof. It is obvious that (2.4.1) is a complex of $R$-modules. We denote by $\bar{x}$ the residue class of an element $x \in S^{n}$ in $R^{n}$. Let $\bar{x}$ be an element of $R^{n}$ with $\phi(\bar{x})=\overline{0}$. Then $\phi(x) \in f S^{n}$, so $\phi(x)=f y$ for some $y \in S^{n}$, and we have $f x=\psi \phi(x)=f \psi(y)$. Since $f$ is an $S$-regular element, we get $x=\psi(y)$, and so $\bar{x}=\psi(\bar{y})$. Therefore $\operatorname{Ker}\left(R^{n} \stackrel{\phi}{\rightarrow} R^{n}\right)=\operatorname{Im}\left(R^{n} \stackrel{\psi}{\rightarrow} R^{n}\right)$. Similarly we obtain $\operatorname{Ker}\left(R^{n} \stackrel{\psi}{\rightarrow} R^{n}\right)=\operatorname{Im}\left(R^{n} \stackrel{\phi}{\rightarrow} R^{n}\right)$. Thus (2.4.1) is an exact sequence. The last statement follows from [8, Theorem (4.1.4)].

Matrix factorizations form a category:

Definition 2.5. We define the category $\mathcal{M}_{S}^{0}(f)$ by setting

(1) the matrix factorizations of $f$ as the objects of $\mathcal{M}_{S}^{0}(f)$, and

(2) a pair $(\alpha, \beta)$ of matrices making the following diagram commute

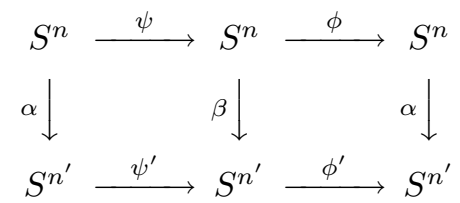

as a morphism from an object $(\phi, \psi)$ to an object $\left(\phi^{\prime}, \psi^{\prime}\right)$.

Remark 2.6. (1) The commutativity of the right square in a diagram of the form (2.5.1) implies the commutativity of the left one. In fact, if $\alpha \phi=\phi^{\prime} \beta$, then $\phi^{\prime}\left(\beta \psi-\psi^{\prime} \alpha\right)=\alpha\left(f I_{n}\right)-\left(f I_{n^{\prime}}\right) \alpha=0$, and $\beta \psi=\psi^{\prime} \alpha$ by the injectivity of $\phi^{\prime}$.

(2) The zero matrix factorization $(\zeta, \zeta)$ is an object of $\mathcal{M}_{S}^{0}(f)$, both terminal and initial, hence zero.

(3) The category $\mathcal{M}_{S}^{0}(f)$ is an additive category. Indeed, for two matrix factorizations $(\phi, \psi)$ and $\left(\phi^{\prime}, \psi^{\prime}\right)$,

$$
(\phi, \psi) \oplus\left(\phi^{\prime}, \psi^{\prime}\right)=\left(\left(\begin{array}{cc}
\phi & 0 \\
0 & \psi
\end{array}\right),\left(\begin{array}{cc}
\phi^{\prime} & 0 \\
0 & \psi^{\prime}
\end{array}\right)\right) .
$$

Definition 2.7. (1) We say that two matrix factorizations $(\phi, \psi),\left(\phi^{\prime}, \psi^{\prime}\right)$ are equivalent, and denote this situation by $(\phi, \psi) \sim\left(\phi^{\prime}, \psi^{\prime}\right)$, if there is an isomorphism $(\phi, \psi) \rightarrow\left(\phi^{\prime}, \psi^{\prime}\right)$ in $\mathcal{M}_{S}^{0}(f)$.

(2) We say that a matrix factorization $(\phi, \psi)$ is reduced if all entries of the matrices $\phi, \psi$ are in $\mathfrak{n}$.

Remark 2.8. (1) Every matrix factorization equivalent to a reduced one is reduced.

(2) The pairs $(1, f),(f, 1)$ of elements of $S$ are always non-reduced matrix factorizations of $f$.

Let $\mathcal{A}$ be an additive category and $\mathcal{B}$ a set of objects of $\mathcal{A}$. Then the category $\mathcal{A} / \mathcal{B}$ has $\operatorname{Ob}(\mathcal{A} / \mathcal{B})=$ $\operatorname{Ob}(\mathcal{A})$ and $\operatorname{Hom}_{\mathcal{A} / \mathcal{B}}\left(A_{1}, A_{2}\right)=\operatorname{Hom}_{\mathcal{A}}\left(A_{1}, A_{2}\right) / \mathcal{B}\left(A_{1}, A_{2}\right)$ for $A_{1}, A_{2} \in \operatorname{Ob}(\mathcal{A} / \mathcal{B})$, where $\mathcal{B}\left(A_{1}, A_{2}\right)$ is the subgroup consisting of all morphisms from $A_{1}$ to $A_{2}$ that factor through finite direct sums of objects in $\mathcal{B}$. Note that $\mathcal{A} / \mathcal{B}$ is also an additive category.

Definition 2.9. We define the following additive categories:

$$
\begin{aligned}
\mathcal{M}_{S}(f) & =\mathcal{M}_{S}^{0}(f) /\{(1, f)\}, \\
\underline{\mathcal{M}}_{S}(f) & =\mathcal{M}_{S}(f) /\{(f, 1)\}=\mathcal{M}_{S}^{0}(f) /\{(1, f),(f, 1)\}, \\
\underline{\mathcal{G}}(R) & =\mathcal{G}(R) /\{R\} .
\end{aligned}
$$

Note that $\underline{\mathcal{G}}(R)$ is the stable category of $\mathcal{G}(R)$.

The following theorem is the main result of this section, which is a generalization of Eisenbud's matrix factorization theorem [10, Section 6] (see also [21, Theorem (7.4)]).

Theorem 2.10. There are equivalences of categories:

$$
\begin{aligned}
& \mathcal{M}_{S}(f) \simeq \mathcal{G}(R), \\
& \mathcal{M}_{S}(f) \simeq \underline{\mathcal{G}}(R) .
\end{aligned}
$$


Proof. For a matrix factorization $(\phi, \psi)$ of $f$, the module $F((\phi, \psi)):=$ Coker $\phi$ is in $\mathcal{G}(R)$ by Proposition 2.4. For a morphism $(\alpha, \beta):(\phi, \psi) \rightarrow\left(\phi^{\prime}, \psi^{\prime}\right)$ of matrix factorizations of $f$, let $F((\alpha, \beta))$ be the induced homomorphism $F((\phi, \psi)) \rightarrow F\left(\left(\phi^{\prime}, \psi^{\prime}\right)\right)$. We obtain an additive functor $F: \mathcal{M}_{S}(f) \rightarrow \mathcal{G}(R)$. Compare this with [21, Proposition (7.2) and Theorem (7.4)].

Let $M$ be a totally reflexive $R$-module. Then we have

$$
0 \geq \operatorname{Gdim}_{R} M=\operatorname{Gdim}_{S /(f)} M=\operatorname{Gdim}_{S} M-1=\operatorname{pd}_{S} M-1 .
$$

Here, the second equality follows from the fact that $f$ is an $S$-regular element in $\operatorname{Ann}_{S} M$ and Lemma 1.5(3), and the third follows by Proposition 1.8(2). Hence the $S$-module $M$ has projective dimension at most one, and there exists an exact sequence $0 \rightarrow S^{n} \stackrel{\phi}{\rightarrow} S^{m} \rightarrow M \rightarrow 0$. By Proposition 2.3(2), we have $n=m$ and there is a matrix $\psi$ such that $(\phi, \psi)$ is a matrix factorization of $f$. By analogous arguments to the proof of [21, Theorem (7.4)], we obtain an additive functor $G: \mathcal{G}(R) \rightarrow \mathcal{M}_{S}(f)$ with $G(M)=(\phi, \psi)$, and see that $F G=1_{\mathcal{G}(R)}$ and $G F \cong 1_{\mathcal{M}_{S}(f)}$. Thus $F$ forms an equivalence between the additive categories $\mathcal{M}_{S}(f)$ and $\mathcal{G}(R)$. Since $F((f, 1))=R$, the functor $F$ induces an additive functor $\underline{\mathcal{M}}_{S}(f) \rightarrow \underline{\mathcal{G}}(R)$ of additive categories which is an equivalence.

The above theorem yields the following corollary; in the case where $R$ is henselian, one can uniquely decompose a given matrix factorization into a direct sum of the form in the corollary. One can prove the corollary similarly to the arguments in [21, Remark (7.5)]. The henselian property of $R$ is used in showing the uniqueness of the direct sum decomposition of $R$-modules induced from (2.11.1) along the first equivalence in Theorem 2.10.

Corollary 2.11. Suppose that $R$ is henselian. Then every matrix factorization $(\phi, \psi)$ of $f$ has a direct sum decomposition unique up to similarity

$$
(\phi, \psi) \sim\left(\phi_{0}, \psi_{0}\right) \oplus(1, f)^{\oplus p} \oplus(f, 1)^{\oplus q},
$$

where $\left(\phi_{0}, \psi_{0}\right)$ is a reduced matrix factorization and $p, q$ are nonnegative integers.

To prove our next result, we establish a lemma.

Lemma 2.12. Let $(\phi, \psi)$ be a matrix factorization of $f$. Assume that $\psi$ has an entry which is a unit of $S$. Then $(\phi, \psi)$ has a direct summand equivalent to $(f, 1)$.

Proof. By assumption, there is a commutative diagram

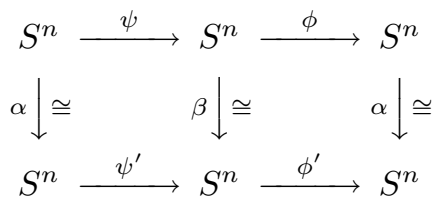

such that $\psi^{\prime}$ is a matrix of the form $\left(\begin{array}{ll}1 & 0 \\ 0 & \nu\end{array}\right)$ and that the vertical maps are isomorphisms. We can directly check that $\left(\phi^{\prime}, \psi^{\prime}\right)$ is a matrix factorization of $f$, and $(\alpha, \beta):(\phi, \psi) \rightarrow\left(\phi^{\prime}, \psi^{\prime}\right)$ is an isomorphism in $\mathcal{M}_{S}^{0}(f)$. Writing $\phi^{\prime}=\left(\begin{array}{ll}a & b \\ c & \mu\end{array}\right)$ and using the equalities $\phi^{\prime} \psi^{\prime}=\psi^{\prime} \phi^{\prime}=f I_{n}$, we see that $a=f, b=0$ and $c=0$, and that $(\mu, \nu)$ is a matrix factorization of $f$. We obtain $(\phi, \psi) \sim\left(\phi^{\prime}, \psi^{\prime}\right)=\left(\left(\begin{array}{ll}f & 0 \\ 0 & \mu\end{array}\right),\left(\begin{array}{ll}1 & 0 \\ 0 & \nu\end{array}\right)\right)=(f, 1) \oplus(\mu, \nu)$, which proves the lemma.

Now we can prove the following proposition. (Note that we do not assume that the local ring $R$ is henselian.)

Proposition 2.13. (cf. [10, Corollary 6.3] and [21, Corollary (7.6)]) The assignment $[(\phi, \psi)] \mapsto[$ Coker $\phi]$ makes a bijection from the set of equivalence classes of reduced matrix factorizations of $f$ to the set of isomorphism classes of totally reflexive $R$-modules without free summand.

Proof. Let $(\phi, \psi)$ be a reduced matrix factorization of $f$. Then Proposition 2.4 and similar arguments to the proof of $[21,(7.5 .1)]$ show that Coker $\phi$ is a totally reflexive $R$-module without free summand. If $(\phi, \psi)$ is equivalent to another reduced matrix factorization $\left(\phi^{\prime}, \psi^{\prime}\right)$ of $f$, then the $R$-module Coker $\phi$ is isomorphic to Coker $\phi^{\prime}$. Thus we obtain a well-defined map

$$
\chi:[(\phi, \psi)] \mapsto[\text { Coker } \phi]
$$


from the set of equivalence classes of reduced matrix factorizations of $f$ to the set of isomorphism classes of totally reflexive $R$-modules without free summand.

Let $(\phi, \psi),\left(\phi^{\prime}, \psi^{\prime}\right)$ be reduced matrix factorizations such that Coker $\phi$ is isomorphic to Coker $\phi^{\prime}$. Then by Proposition $2.3(2)$ we have a commutative diagram

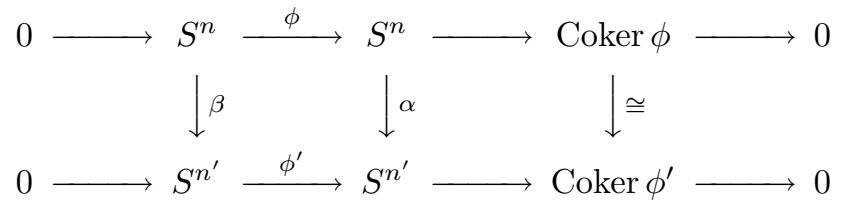

of $S$-modules with exact rows. Since all entries of $\phi, \phi^{\prime}$ are nonunits of $S$, the two rows are minimal free resolutions of the $S$-modules Coker $\phi$, Coker $\phi^{\prime}$. Hence the vertical maps $\alpha, \beta$ are isomorphisms (cf. [12, $\S 18$, Lemma 8]). According to Remark 2.6(1), we have an isomorphism $(\alpha, \beta):(\phi, \psi) \rightarrow\left(\phi^{\prime}, \psi^{\prime}\right)$ in the category $\mathcal{M}_{S}^{0}(f)$. Thus the map $\chi$ is injective.

Let $M$ be a totally reflexive $R$-module. Then it is seen from the proof of Theorem 2.10 that there exists an exact sequence $0 \rightarrow S^{n} \stackrel{\phi}{\rightarrow} S^{n} \rightarrow M \rightarrow 0$ of $S$-modules. We can choose $\phi$ such that all the entries of $\phi$ are in the maximal ideal $\mathfrak{n}$ of $S$. Proposition 2.3(2) shows that there is a matrix $\psi$ such that $(\phi, \psi)$ is a matrix factorization of $f$. By Lemma 2.12 if $M$ has no free $R$-summand, all the entries of the matrix $\psi$ must be in $\mathfrak{n}$. Therefore when $M$ is without free $R$-summand, $(\phi, \psi)$ is a reduced matrix factorization of $f$ such that Coker $\phi=M$. Thus, the map $\chi$ is surjective. This completes the proof of the proposition.

We end this section by mentioning extensions of totally reflexive modules:

Remark 2.14. (cf. [21, Remark (7.8)])

(1) Let $h: M \rightarrow M^{\prime}$ be a homomorphism of totally reflexive $R$-modules. Then the following hold.

(i) There is a morphism $(\alpha, \beta):(\phi, \psi) \rightarrow\left(\phi^{\prime}, \psi^{\prime}\right)$ in $\mathcal{M}_{S}^{0}(f)$ which induces $h$.

(ii) One has a matrix factorization $\left(\left(\begin{array}{cc}\psi^{\prime} & \beta \\ 0 & \phi\end{array}\right),\left(\begin{array}{cc}\phi^{\prime} & -\alpha \\ 0 & \psi\end{array}\right)\right)$ of $f$.

(iii) For the exact sequence $0 \rightarrow N \rightarrow R^{n^{\prime}} \rightarrow M^{\prime} \rightarrow 0$ with $N=$ Coker $\psi^{\prime}$, the connecting homomorphism $\operatorname{Hom}_{R}\left(M, M^{\prime}\right) \rightarrow \operatorname{Ext}_{R}^{1}(M, N)$ sends $h$ to an element corresponding to an exact sequence $0 \rightarrow N \rightarrow L \rightarrow M \rightarrow 0$ with $L=\operatorname{Coker}\left(\begin{array}{cc}\psi^{\prime} & \beta \\ 0 & \phi\end{array}\right)$.

(2) Let $M, N$ be totally reflexive $R$-modules. Every extension $0 \rightarrow N \rightarrow L \rightarrow M \rightarrow 0$ of $M$ by $N$ is obtained in the way shown in (1).

\section{KNÖRRER's PERIODICITY}

In this section, we extend the concept of Knörrer's periodicity [11]. Throughout this section, as in the previous section, let $S$ be a G-regular local ring with maximal ideal $\mathfrak{n}, f \in \mathfrak{n}$ an $S$-regular element, and $R=S /(f)$ the residue ring. Set

$$
A=S[[x]] /\left(f+x^{2}\right) \text { and } B=S[[x, y]] /(f+x y),
$$

where $x, y$ are indeterminates over $S$.

We can directly check that the following statements hold.

(1) One has $A /(x) \cong R$.

(2) The element $x$ is $A$-regular.

(3) The ring $A$ is a free $S$-module with basis $\{1, x\}$.

(4) The element $f+x^{2}$ is $S[[x]]$-regular.

For a totally reflexive $A$-module $M$, we set $\Theta M=M / x M$.

Proposition 3.1. One has an additive functor $\Theta: \mathcal{G}(A) \rightarrow \mathcal{G}(R)$.

Proof. It is seen from Lemma $1.5(3)$ that $\Theta M$ is a totally reflexive $R$-module for a totally reflexive $A$-module $M$. The proposition follows from this.

Proposition 3.2. An A-module is totally reflexive if and only if it is free as an $S$-module. 
Proof. Let $M$ be a nonzero $A$-module. We have an equality

$$
\operatorname{Gdim}_{A} M=\operatorname{Gdim}_{S[[x]]} M-1
$$

by Lemma 1.5(3).

Suppose that $M$ is free as an $S$-module. Let $m \in M$ with $x m=0$. Then $f m=-x^{2} m=0$. Since $f$ is $S$-regular and $M$ is assumed to be $S$-free, $f$ is $M$-regular. Therefore $m=0$. Nakayama's lemma implies that $x M \neq M$. Thus $x$ is an $M$-regular element. It follows from Lemma $1.5(3)$ that $\operatorname{Gdim}_{S[[x]]} M=\operatorname{Gdim}_{S} M / x M$. There is an exact sequence $0 \rightarrow M \stackrel{x}{\rightarrow} M \rightarrow M / x M \rightarrow 0$, and $M$ is totally reflexive over $S$ since it is free over $S$. This yields an inequality $\operatorname{Gdim}_{S} M / x M \leq 1$, so we have $\operatorname{Gdim}_{A} M \leq 0$ by (3.2.1). Hence $M$ is totally reflexive over $A$.

On the other hand, suppose that $M$ is a totally reflexive $A$-module. Then $\operatorname{Gdim}_{A} M \leq 0$, and $\operatorname{Gdim}_{S[[x]]} M \leq 1$ by (3.2.1). Corollary 4.4 and Proposition 1.8(2) imply that $\operatorname{Gdim}_{S[[x]]} M=\operatorname{pd}_{S[[x]]} M$. Hence $\operatorname{pd}_{S[[x]]} M \leq 1$, and there is an exact sequence

$$
0 \rightarrow F_{1} \rightarrow F_{0} \rightarrow M \rightarrow 0
$$

such that $F_{0}, F_{1}$ are free $S[[x]]$-modules. Note that $F_{0}, F_{1}$ are flat as $S$-modules, so we have $\operatorname{Tor}_{i}^{S}(M, S / \mathfrak{n})=0$ for any $i \geq 2$. The $\operatorname{ring} A$ is finitely generated as an $S$-module, hence so is $M$. It follows that $\operatorname{pd}_{S} M \leq 1<\infty$ (cf. [7, Corollary 1.3.2]). Applying Lemma 1.5(2), we obtain $\operatorname{pd}_{S} M=\operatorname{depth} S-\operatorname{depth}_{S} M$. It is obvious that the closed fiber $A / \mathfrak{n} A$ of the flat local homomorphism $S \rightarrow A$ is artinian. Therefore we have equalities $\operatorname{depth}_{A} M=\operatorname{depth}_{S} M$ and $\operatorname{depth} A=$ $\operatorname{depth} S+\operatorname{depth} A / \mathfrak{n} A=\operatorname{depth} S . \operatorname{Thus}_{\operatorname{pd}_{S}} M=\operatorname{depth} A-\operatorname{depth}_{A} M=\operatorname{Gdim}_{A} M \leq 0$ by Lemma $1.5(2)$, and $M$ is $S$-free.

As a direct consequence of Proposition 3.2, we have the following result.

Corollary 3.3. The totally reflexive A-modules are precisely the free $S$-modules with A-module structure, or equivalently, the free $S$-modules on which $x$ acts.

Recall that two square matrices $\phi, \psi$ over $S$ of the same size are similar if there exists an $n \times n$ invertible matrix $\alpha$ over $S$ such that $\phi=\alpha^{-1} \psi \alpha$. For a totally reflexive $A$-module $M$, we denote by $\phi_{M}$ a representation matrix of the linear map $M \stackrel{x}{\rightarrow} M$ (the multiplication map by the variable $x$ ) of free $S$-modules. Note that $\phi_{M}$ is not uniquely determined by $M$. Instead, we have the following.

Corollary 3.4. The assignment $[M] \mapsto\left[\phi_{M}\right]$ makes a bijection from the set of isomorphism classes of totally reflexive A-modules to the set of similarity classes of square matrices $\phi$ over $S$ with $\phi^{2}=-f I$, where $I$ is the identity matrix.

Proof. Let $M$ be a totally reflexive $A$-module. Then Proposition 3.2 shows that there is a commutative diagram

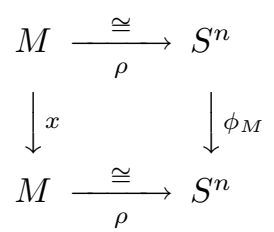

where $\rho$ is an $S$-isomorphism. We have $\phi_{M}=\rho x \rho^{-1}$, and hence $\phi_{M}^{2}=\left(\rho x \rho^{-1}\right)\left(\rho x \rho^{-1}\right)=\rho x^{2} \rho^{-1}=$ $\rho(-f) \rho^{-1}=-f\left(\rho \rho^{-1}\right)=-f I_{n}$.

Let $M$ and $N$ be totally reflexive $A$-modules with $[M]=[N]$. Then there exists an $A$-isomorphism $\lambda: M \rightarrow N$, and we have $x \lambda=\lambda x$. There are $S$-isomorphisms $\rho_{M}: M \rightarrow S^{n}$ and $\rho_{N}: N \rightarrow S^{n}$ such that $\phi_{M} \rho_{M}=\rho_{M} x$ and $\phi_{N} \rho_{N}=\rho_{N} x$. Setting $\alpha=\rho_{N} \lambda \rho_{M}^{-1}$, we easily see that $\alpha$ is an invertible matrix over $S$ satisfying $\alpha^{-1} \phi_{N} \alpha=\phi_{M}$. Therefore $\left[\phi_{M}\right]=\left[\phi_{N}\right]$. Thus, we obtain a well-defined map

$$
\chi:[M] \mapsto\left[\phi_{M}\right]
$$

from the set of isomorphism classes of totally reflexive $A$-modules to the set of similarity classes of square matrices $\phi$ over $S$ with $\phi^{2}=-f I$.

Let $M, N$ be totally reflexive $A$-modules with $\left[\phi_{M}\right]=\left[\phi_{N}\right]$. Then there exists an invertible matrix $\alpha$ over $S$ with $\phi_{M}=\alpha^{-1} \phi_{N} \alpha$. As before, there exist $S$-isomorphisms $\rho_{M}: M \rightarrow S^{n}$ and $\rho_{N}: N \rightarrow S^{n}$ such 
that $\phi_{M} \rho_{M}=\rho_{M} x$ and $\phi_{N} \rho_{N}=\rho_{N} x$. Putting $\lambda=\rho_{N}^{-1} \alpha \rho_{M}$, we have $\lambda x=x \lambda$, which means that $\lambda$ is an $A$-homomorphism, hence an $A$-isomorphism. Thus we have $[M]=[N]$, and the map $\chi$ is injective.

Let $\phi$ be an $n \times n$-matrix over $S$ with $\phi^{2}=-f I_{n}$. Then, letting $M$ be the free $S$-module $S^{n}$ equipped with the action of $x$ by $x z=\phi(z)$ for $z \in M$, we have $x^{2} z=\phi^{2}(z)=-f z$ and we see that $M$ is an $A$-module. Proposition 3.2 says that $M$ is a totally reflexive $A$-module. Since $\phi$ is a representation matrix of the $S$-linear map $M \stackrel{x}{\rightarrow} M$, we have $[\phi]=\left[\phi_{M}\right]$. Thus the map $\chi$ is surjective.

Using Corollary 3.4, we can show the following result along the same lines as in the proof of [21, Lemma (12.2)].

Lemma 3.5. Let $M$ be a totally reflexive A-module. Then the following hold.

(1) One has a matrix factorization $\left(x I-\phi_{M}, x I+\phi_{M}\right)$ of $f+x^{2}$ over $S[[x]]$, and $M \cong \operatorname{Coker}\left(x I-\phi_{M}\right)$.

(2) One has a matrix factorization $\left(\phi_{M},-\phi_{M}\right)$ of $f$ over $S$, and $\Theta M \cong$ Coker $\phi_{M}$.

We have a functor in the opposite direction to that of the functor $\Theta$.

Proposition 3.6. Taking the first syzygy makes an additive functor $\Omega_{A}: \mathcal{G}(R) \rightarrow \mathcal{G}(A)$.

Proof. For a totally reflexive $R$-module $M$, we have $0 \geq \operatorname{Gdim}_{A /(x)} M=\operatorname{Gdim}_{A} M-1$ by Lemma 1.5(3), and $\operatorname{Gdim}_{A}\left(\Omega_{A} M\right) \leq 0$ by Lemma $1.5(4)$. Therefore $\Omega_{A} M$ is a totally reflexive $A$-module.

The following lemma is an analogue of the second statement in Lemma 3.5. We can show it similarly to the proof of [21, Lemma (12.3)] by using Proposition 2.13.

Lemma 3.7. (1) Let $(\phi, \psi)$ be a matrix factorization of $f$ over $S$, and put $M=$ Coker $\phi$. Then $\left(\left(\begin{array}{cc}\psi & -x I \\ x I & \phi\end{array}\right),\left(\begin{array}{cc}\phi & x I \\ -x I & \psi\end{array}\right)\right)$ is a matrix factorization of $f+x^{2}$ over $S[[x]]$, and $\Omega_{A} M \oplus F \cong \operatorname{Coker}\left(\begin{array}{cc}\psi & -x I \\ x I & \phi\end{array}\right)$ for some free $A$-module $F$.

(2) In (1), assume in addition that $(\phi, \psi)$ is reduced. Then $\left(\left(\begin{array}{cc}\psi & -x I \\ x I & \phi\end{array}\right),\left(\begin{array}{cc}\phi & x I \\ -x I & \psi\end{array}\right)\right)$ is also reduced, and $\Omega_{A} M \cong \operatorname{Coker}\left(\begin{array}{cc}\psi & -x I \\ x I & \phi\end{array}\right)$.

Remark 3.8. Let $(\phi, \psi)$ be a reduced matrix factorization of $f$ over $S$ and set $M=$ Coker $\phi$. Then one has an equality

$$
\left(\begin{array}{cc}
0 & I \\
-I & 0
\end{array}\right)\left(\begin{array}{cc}
\psi & -x I \\
x I & \phi
\end{array}\right)\left(\begin{array}{cc}
0 & -I \\
I & 0
\end{array}\right)=\left(\begin{array}{cc}
\phi & -x I \\
x I & \psi
\end{array}\right),
$$

which shows that Coker $\left(\begin{array}{cc}\psi & -x I \\ x I & \phi\end{array}\right)$ is isomorphic to Coker $\left(\begin{array}{cc}\phi & -x I \\ x I & \psi\end{array}\right)$. Therefore it follows from Lemma $3.7(2)$ that the $A$-module $\Omega_{A} \Omega_{R} M$ is isomorphic to $\Omega_{A} M$.

Applying Lemmas 3.5 and 3.7 and Proposition 2.13, one can prove the following result along the same lines as in the proof of [21, Proposition (12.4)].

Proposition 3.9. (1) For a totally reflexive $R$-module $M$ without free summand, one has $\Theta \Omega_{A} M \cong$ $M \oplus \Omega_{R} M$.

(2) Assume that $\frac{1}{2} \in S$. Then for a totally reflexive $A$-module $N$, one has $\Omega_{A} \Theta N \cong N \oplus \Omega_{A} N$ up to free summand.

Corollary 3.10. (cf. [21, Remark (12.7)]) Suppose that $R$ is henselian. Then the following hold.

(1) (i) For any nonfree indecomposable totally reflexive $R$-module $M$, there exists a nonfree indecomposable totally reflexive $A$-module $N$ such that $M$ is isomorphic to a direct summand of $\Theta N$.

(ii) Assume that $\frac{1}{2} \in S$. Then for any nonfree indecomposable totally reflexive A-module $N$, there exists a nonfree indecomposable totally reflexive $R$-module $M$ such that $N$ is isomorphic to a direct summand of $\Omega_{A} M$.

(2) For an indecomposable totally reflexive $R$-module $M$, the $A$-module $\Omega_{A} M$ has at most two nonzero direct summands.

Proof. The assertion (1) follows from Proposition 3.9 and analogous arguments to the proof of [21, Theorem (12.5)]. As to the assertion (2), we may assume that the $R$-module $M$ is nonfree, hence $M$ has no free summand. Suppose that there is a direct sum decomposition $\Omega_{A} M \cong X \oplus Y \oplus Z$ of $A$ modules. Then we have $\Theta X \oplus \Theta Y \oplus \Theta Z \cong \Theta \Omega_{A} M \cong M \oplus \Omega_{R} M$ by Proposition 3.9(1). According to [16, Proposition 7.1], $\Omega_{R} M$ is also indecomposable. By virtue of the Krull-Schmidt theorem, one of the $R$-modules $\Theta X, \Theta Y, \Theta Z$ is zero; we may assume that $\Theta Z=0$. Then we have $x Z=Z$, and $Z=0$ by Nakayama's lemma. This shows the assertion (2). 
For a matrix factorization $(\phi, \psi)$ of $f$ over $S$, set

$$
\Delta^{0}(\phi, \psi)=\left(\left(\begin{array}{cc}
\phi & x I \\
y I & -\psi
\end{array}\right),\left(\begin{array}{cc}
\psi & x I \\
y I & -\phi
\end{array}\right)\right) .
$$

Note that this is a matrix factorization of $f+x y$ over $S[[x, y]]$. For a morphism $(\alpha, \beta):(\phi, \psi) \rightarrow\left(\phi^{\prime}, \psi^{\prime}\right)$ in the category $\mathcal{M}_{S}^{0}(f)$ of matrix factorizations of $f$ over $S$, let

$$
\Delta^{0}(\alpha, \beta)=\left(\left(\begin{array}{cc}
\alpha & 0 \\
0 & \beta
\end{array}\right),\left(\begin{array}{ll}
\beta & 0 \\
0 & \alpha
\end{array}\right)\right) .
$$

Note that this is a morphism in $\mathcal{M}_{S[[x, y]]}^{0}(f+x y)$. Thus we obtain an additive functor

$$
\Delta^{0}: \mathcal{M}_{S}^{0}(f) \rightarrow \mathcal{M}_{S[[x, y]]}^{0}(f+x y)
$$

Since there is a commutative diagram

$$
\begin{aligned}
& S[[x, y]]^{2} \stackrel{\left(\begin{array}{ll}
1 & x \\
y & -f
\end{array}\right)}{\longrightarrow} S[[x, y]]^{2} \stackrel{\left(\begin{array}{cc}
f & x \\
y & -1
\end{array}\right)}{\longrightarrow} S[[x, y]]^{2} \\
& \cong \downarrow\left(\begin{array}{ll}
1 & x \\
0 & 1
\end{array}\right) \quad \cong \downarrow\left(\begin{array}{cc}
1 & 0 \\
y & -1
\end{array}\right) \quad \cong \downarrow\left(\begin{array}{ll}
1 & x \\
0 & 1
\end{array}\right) \\
& S[[x, y]]^{2} \stackrel{\left(\begin{array}{ll}
1 & 0 \\
0 & f+x y
\end{array}\right)}{\longrightarrow} S[[x, y]]^{2} \stackrel{\left(\begin{array}{c}
f+x y \\
0
\end{array}\right)}{\longrightarrow} S[[x, y]]^{2}
\end{aligned}
$$

with isomorphic vertical maps, both $\Delta^{0}(f, 1)$ and $\Delta^{0}(1, f)$ are isomorphic to $(f+x y, 1) \oplus(1, f+x y)$. Hence $\Delta^{0}$ induces an additive functor

$$
\Delta: \underline{\mathcal{M}}_{S}(f) \rightarrow \underline{\mathcal{M}}_{S[[x, y]]}(f+x y) .
$$

By virtue of Theorem 2.10, we get an additive functor

$$
\underline{\mathcal{G}}(R) \rightarrow \underline{\mathcal{G}}(B) .
$$

We also denote it by $\Delta$.

The same proof as that of [21, Lemma (12.9)] shows the following result.

Lemma 3.11. Let

$$
\left(\left(\begin{array}{ll}
a & b \\
c & d
\end{array}\right),\left(\begin{array}{ll}
a^{\prime} & b^{\prime} \\
c^{\prime} & d^{\prime}
\end{array}\right)\right):\left(\left(\begin{array}{cc}
\phi & x \\
y & -\psi
\end{array}\right),\left(\begin{array}{cc}
\psi & x \\
y & -\phi
\end{array}\right)\right) \rightarrow\left(\left(\begin{array}{cc}
\phi^{\prime} & x \\
y & -\psi^{\prime}
\end{array}\right),\left(\begin{array}{cc}
\psi^{\prime} & x \\
y & -\phi^{\prime}
\end{array}\right)\right)
$$

be a morphism in $\mathcal{M}_{S[[x, y]]}^{0}(f+x y)$. Assume that all the entries of the matrix $a^{\prime}$ are in the maximal ideal $(x, y) S[[x, y]]$ of the local ring $S[[x, y]]$. Then one has an equivalence

$$
\left(\left(\begin{array}{cccc}
\psi^{\prime} & x & a^{\prime} & b^{\prime} \\
y & -\phi^{\prime} & c^{\prime} & d^{\prime} \\
0 & 0 & \phi & x \\
0 & 0 & y & -\psi
\end{array}\right),\left(\begin{array}{cccc}
\phi^{\prime} & x & -a & -b \\
y & -\psi^{\prime} & -c & -d \\
0 & 0 & \psi & x \\
0 & 0 & y & -\phi
\end{array}\right)\right) \sim\left(\left(\begin{array}{cc}
\psi^{\prime} & x \\
y & -\phi^{\prime}
\end{array}\right),\left(\begin{array}{cc}
\phi^{\prime} & x \\
y & -\psi^{\prime}
\end{array}\right)\right) \oplus\left(\left(\begin{array}{cc}
\phi & x \\
y & -\psi
\end{array}\right),\left(\begin{array}{cc}
\psi & x \\
y & -\phi
\end{array}\right)\right)
$$

of matrix factorizations of $f+x y$ over $S[[x, y]]$.

The theorem below is the main result of this section, which is a generalized version of Knörrer's periodicity theorem [11, Theorem 3.1].

Theorem 3.12. (1) The functor $\Delta: \underline{\mathcal{G}}(R) \rightarrow \underline{\mathcal{G}}(B)$ is fully faithful.

(2) Suppose that $\frac{1}{2}, \sqrt{-1} \in S$ and that $R$ is henselian. Then the functor $\Delta: \underline{\mathcal{G}}(R) \rightarrow \underline{\mathcal{G}}(B)$ is an equivalence.

Proof. Both of the assertions can be proved similarly to the proof of [21, Theorem (12.10)]. For the first assertion, we use Remark 2.14 and Lemma 3.11. As to the second assertion, note from the assumption that $B=S[[x, y]] /(f+x y)=S[[u, v]] /\left(f+u^{2}+v^{2}\right)$ where $u=\frac{x+y}{2}, v=\frac{x-y}{2 \sqrt{-1}}$. Apply Proposition 3.9, Corollary 3.10 and Lemma 3.7.

By analogous arguments to the proof of [21, Corollary (12.11)] and Proposition 2.13, we obtain a corollary of Theorem 3.12.

Corollary 3.13. Suppose that $R$ is henselian.

(1) Let $g: M \rightarrow N$ be a homomorphism of totally reflexive $R$-modules such that $M$ is nonfree and indecomposable. Then $g$ is a split monomorphism (respectively, split epimorphism) if and only if so is $\Delta^{0} g$.

(2) Assume that $\frac{1}{2}, \sqrt{-1} \in S$. Let $M$ be a nonfree indecomposable totally reflexive $R$-module. Then $\Delta^{0} M$ is a nonfree indecomposable totally reflexive $B$-module. 


\section{The ASCENT AND DESCENT OF G-REgUlar PROPERTY}

We investigate ascent and descent of the G-regular property, modeling our study on the situations where ascent and descent of the regular property is known to hold. First, the G-regular property descends through flat local homomorphisms.

Proposition 4.1. Let $R \rightarrow S$ be a flat local ring homomorphism. If $S$ is G-regular, then so is $R$.

Proof. Let $M$ be a totally reflexive $R$-module. Then $M \otimes_{R} S$ is a totally reflexive $S$-module by [4, Theorem 8.7(6)]. Since $S$ is G-regular, $M \otimes_{R} S$ is a free $S$-module. Applying [4, Theorem 8.7(6)] again, we see that $M$ is a free $R$-module. Thus $R$ is also G-regular.

Proposition 4.2. Let $R$ be a local ring and $\boldsymbol{x}=x_{1}, \ldots, x_{n}$ an $R$-sequence. If $R /(\boldsymbol{x})$ is $G$-regular, then so is $R$.

Proof. We may assume that $n=1$. Let $M$ be a totally reflexive $R$-module. According to Lemma 1.5(3), $M / x_{1} M$ is a totally reflexive $R /\left(x_{1}\right)$-module, and so it is a free $R /\left(x_{1}\right)$-module by assumption. The $R$-module $M$ is torsionfree since it is reflexive, so $x_{1}$ is an $M$-regular element. By [7, Lemma 1.3.5], $M$ is a free $R$-module. It follows that $R$ is a G-regular local ring.

Remark 4.3. The converse of Proposition 4.2 does not necessarily hold. In fact, let $k$ be a field and let $R=k[[t]]$ be a formal power series ring. Then $R$ is regular, so $R$ is G-regular by Proposition 1.8(1). The element $t^{2}$ of $R$ is $R$-regular. However, since $R /\left(t^{2}\right)=k[[t]] /\left(t^{2}\right)$ is a singular Gorenstein local ring, it is not G-regular by Proposition 1.8(1) again.

Corollary 4.4. Let $n$ be a positive integer. A local ring $R$ is G-regular if and only if so is the formal power series ring $R\left[\left[X_{1}, \ldots, X_{n}\right]\right]$.

Proof. The "if" part follows from Proposition 4.1, and the "only if" part follows from Proposition 4.2.

Corollary 4.5. Let $R \rightarrow S$ be a flat local ring homomorphism, and let $\mathfrak{m}$ denote the unique maximal ideal of $R$. If $R$ is regular and $S / \mathfrak{m} S$ is G-regular, then $S$ is also G-regular.

Proof. Let $\boldsymbol{x}=x_{1}, \ldots, x_{d}$ be a regular system of parameters of $R$. The residue $\operatorname{ring} S / \boldsymbol{x} S=S / \mathfrak{m} S$ is a G-regular local ring. Since $S$ is flat over $R$, the sequence $\boldsymbol{x}$ is $S$-regular. It follows from Proposition 4.2 that $S$ is G-regular.

Proposition 4.6. Let $(R, \mathfrak{m})$ be a $G$-regular local ring and $x \in \mathfrak{m}$ an $R$-regular element. Then $R /(x)$ is $G$-regular if and only if $x \notin \mathfrak{m}^{2}$.

Proof. The "if" part: Suppose that $R /(x)$ is not G-regular. Then there exists a nonfree totally reflexive $R /(x)$-module $N$. We can assume without loss of generality that $N$ is indecomposable. Hence $N$ has no free $R /(x)$-summand. Proposition 2.13 implies that there is a reduced matrix factorization $(\phi, \psi)$ of $x$ over $R$ such that Coker $\phi=N$. Thus all the entries of the matrices $\phi, \psi$ are in the maximal ideal $\mathfrak{m}$ of $R$. The equality $\phi \psi=x I$, where $I$ is the identity matrix, shows that $x$ is an element in $\mathfrak{m}^{2}$.

The "only if" part: Suppose that $x \in \mathfrak{m}^{2}$. Then one can write $x=\sum_{i=1}^{r} y_{i} z_{i}$ for some $r \geq 1$ and $y_{i}, z_{i} \in \mathfrak{m}$. Let $e_{1}, \ldots, e_{r}$ be the canonical basis of the free $R$-module $F:=R^{r}$. We define two $R$-linear maps $\mu, \nu$ from the exterior algebra $\bigwedge F$ of $F$ to itself by

$$
\begin{aligned}
\mu\left(e_{i_{1}} \wedge \cdots \wedge e_{i_{s}}\right) & =\sum_{j=1}^{s}(-1)^{j-1} y_{i_{j}}\left(e_{i_{1}} \wedge \cdots \wedge e_{i_{j-1}} \wedge e_{i_{j+1}} \wedge \cdots \wedge e_{i_{s}}\right), \\
\nu(w) & =\left(\sum_{j=1}^{r} z_{j} e_{j}\right) \wedge w .
\end{aligned}
$$

Note that $\bigwedge F$ is a free $R$-module of rank $2^{r}$. Setting $\phi=\mu+\nu$, we see that $(\phi, \phi)$ is a matrix factorization of $x$ over $R$; see [21, Lemma (8.14)]. Since the images of $\mu, \nu$ are contained in the maximal ideal $\mathfrak{m}$, the image of the $R$-linear map $\phi$ are contained in $\mathfrak{m}(\bigwedge F)$, namely, the matrix factorization $(\phi, \phi)$ is reduced. It follows by Proposition 2.13 that Coker $\phi$ is a nonfree totally reflexive $R /(x)$-module. Hence $R /(x)$ is not a G-regular local ring. 
Corollary 4.7. A local ring $(R, \mathfrak{m})$ is $G$-regular if and only if so is its $\mathfrak{m}$-adic completion $\widehat{R}$.

Proof. The "if" part follows from Proposition 4.1. Let us show the "only if" part; suppose that $R$ is a G-regular local ring. Let $x_{1}, \ldots, x_{n}$ be a system of generators of the maximal ideal $\mathfrak{m}$ of $R$. Then there is an isomorphism

$$
\widehat{R} \cong R\left[\left[X_{1}, \ldots, X_{n}\right]\right] /\left(X_{1}-x_{1}, \ldots, X_{n}-x_{n}\right),
$$

where $X_{1}, \ldots, X_{n}$ are indeterminates over $R$. Corollary 4.4 and Proposition 4.6 imply that the local ring $\widehat{R}$ is G-regular.

\section{SufFicIENT CONDITIONS FOR G-REGUlAR PROPERTY}

In this section, we give some sufficient conditions for a given local ring to be G-regular. We also construct several examples of G-regular local rings.

A sufficient condition is given by the following result, which was proved by Avramov and Martsinkovsky [5, Examples 3.5]. See also [22, Corollary 2.5].

Lemma 5.1. Every Golod local ring which is not a hypersurface is G-regular. In particular, every non-Gorenstein Cohen-Macaulay local ring with minimal multiplicity is G-regular.

Example 5.2. According to Lemma 5.1, for examples, the local algebras

$$
k[[x, y]] /\left(x^{2}, x y, y^{2}\right), \quad k[[x, y, z]] /\left(x^{2}-y z, y^{2}-x z, z^{2}-x y\right), \quad k\left[\left[t^{3}, t^{4}, t^{5}\right]\right](\subseteq k[[t]])
$$

over a field $k$, where $x, y, z, t$ are indeterminates over $k$, are G-regular, since all of them are non-Gorenstein Cohen-Macaulay local rings with minimal multiplicity.

In the above example, the first ring shows that a G-regular local ring is not necessarily a domain, while every regular local ring is a domain.

The following result is due to Yoshino [22, Theorem 3.1]. Using its contrapositive we obtain some sufficient conditions for a local ring to be G-regular.

Lemma 5.3. Let $(R, \mathfrak{m})$ be a non-Gorenstein local ring with $\mathfrak{m}^{3}=0 \neq \mathfrak{m}^{2}$ with a coefficient field $k$. Suppose that $R$ is not G-regular. Then $R$ is a standard graded Koszul k-algebra, and the Hilbert series $H_{R}(t)$ of the ring $R$, the Poincaré series $P_{R / \mathfrak{m}}(t)$ of the $R$-module $R / \mathfrak{m}$, and the Bass series $I^{R}(t)$ of the $R$-module $R$ are as follows:

$$
H_{R}(t)=1+(r+1) t+r t^{2}, \quad P_{R / \mathfrak{m}}(t)=\frac{1}{1-(r+1) t+r t^{2}}, \quad I^{R}(t)=\frac{r-t}{1-r t} .
$$

Here, $r=\operatorname{dim}_{k} \operatorname{Hom}_{R}(k, R)$ is the type of $R$.

Taking advantage of this lemma, we can construct G-regular local rings that do not have minimal multiplicity.

Example 5.4. Let $k$ be a field.

(1) The ring

$$
R=k[[x, y]] /\left(x^{3}, x y, y^{3}\right)
$$

is an artinian non-Gorenstein G-regular local ring which does not have minimal multiplicity by the Hilbert series computation of Lemma 5.3.

(2) The ring

$$
S=k[[x, y, z]] /\left(x^{3}-y^{2} z, y^{3}-x^{2} z, z^{2}-x y\right)
$$

is a 1-dimensional Cohen-Macaulay non-Gorenstein G-regular local ring not having minimal multiplicity. Indeed, $S / z S=R$ is a G-regular local ring by (1), and $z$ is a nonzerodivisor of $S$. Hence Proposition 4.2 shows that the local ring $S$ is also G-regular.

The G-regular local rings constructed above are all Cohen-Macaulay. Now, let us construct an example of a non-Cohen-Macaulay G-regular local ring. 
Example 5.5. Let us consider the local algebra

$$
R=k[[x, y]] /\left(x^{2}, x y\right)
$$

over a field $k$. This is a non-Cohen-Macaulay G-regular local ring.

In fact, suppose that $R$ is not G-regular. Then there exists a nonfree totally reflexive $R$-module $M$. We can assume without loss of generality that $M$ is indecomposable. The first syzygy $N=\Omega_{R} M$ of $M$ is also a nonfree indecomposable totally reflexive $R$-module by [16, Proposition 7.1]. Note that there is a free $R$-module $F$ such that $N$ is contained in $\mathfrak{m} F$. Hence we have $x N \subseteq x \mathfrak{m} F=\left(x^{2}, x y\right) F=0$. Thus $N$ is an $R /(x)$-module. Since $R /(x)=k[[y]]$ is a principal ideal domain, the structure theorem (for finitely generated modules over a principal ideal domain) and the indecomposability of $N$ show that $N$ is isomorphic as an $R /(x)$-module to either $R /(x)$ or $R /\left(x, y^{n}\right)$ for some $n \geq 1$. But there is an exact sequence

$$
0 \rightarrow k \rightarrow R \rightarrow R /(x) \rightarrow 0,
$$

which implies that the $R$-module $R /(x)$ is of infinite G-dimension by Lemma 1.5(4) and (1). Also, we have $\operatorname{Hom}_{R}\left(R /\left(x, y^{n}\right), R\right) \cong\left(0:_{R}\left(x, y^{n}\right)\right)=(x) \cong k$, which implies that $R /\left(x, y^{n}\right)$ is not a reflexive $R$-module for any $n \geq 2$. When $n=1$, we have $R /\left(x, y^{n}\right)=k$, which has infinite G-dimension as an $R$-module by Lemma 1.5(1). Since the $R$-module $N$ is totally reflexive, we get a contradiction, and we conclude that $R$ is a G-regular local ring.

\section{Some PROBLEMS}

Question 6.1. Let $R \rightarrow S$ be a flat local homomorphism of local rings. Suppose that both $R$ and $S / \mathfrak{m} S$ are G-regular, where $\mathfrak{m}$ is the maximal ideal of $R$. Then is $S$ also G-regular?

Partial answers to the above question have been obtained in Corollaries 4.4, 4.5 and 4.7.

It is natural to ask if a localization of a G-regular local ring at a prime ideal is G-regular or not. This does not have an affirmative answer in general, as we see in the following example.

Example 6.2. Let $k$ be a field, and let

$$
R=k[[x, y, z]] /\left(x^{2}, x z, y z\right) .
$$

The element $y-z$ is a nonzerodivisor of $R$, and we have $\mathfrak{m}^{2}=(y-z) \mathfrak{m}$. Hence $R$ is a 1 -dimensional Cohen-Macaulay non-Gorenstein local ring with minimal multiplicity, so $R$ is G-regular by Lemma 5.1. Localizing $R$ at the prime ideal $\mathfrak{p}=(x, z)$, we have

$$
R_{\mathfrak{p}} \cong k[[x, y]]_{(x)} /\left(x^{2}\right),
$$

which is a singular Gorenstein local ring. Proposition 1.8(1) says that $R_{\mathfrak{p}}$ is not G-regular.

Let $R$ be a local ring. An $R$-module $M$ is called bounded if the set of the Betti numbers of $M$ admits an upper bound. An $R$-module $M$ is said to be periodic of period $n$, where $n$ is a positive integer, if the $n$th syzygy $\Omega_{R}^{n} M$ is isomorphic to $M$. We just say that $M$ is periodic if $M$ is either free or periodic of period $n$ for some integer $n \geq 1$. We say that $M$ is eventually periodic if there exists an integer $r \geq 0$ such that $\Omega_{R}^{r} M$ is periodic.

A well-known theorem of Eisenbud [10] asserts that every bounded module over a complete intersection local ring is eventually periodic of period 2 . To be precise, let $S$ be a regular local ring, $\boldsymbol{x}=x_{1}, \ldots, x_{n}$ an $S$-sequence, and $R=S /(\boldsymbol{x})$ the residue ring. Then Eisenbud's theorem says that any bounded $R$-module in $\mathcal{C}(R)$ is eventually periodic of period 2. The following question asks whether the G-regular version of this result holds.

Question 6.3. Let $S$ be a G-regular local ring, $\boldsymbol{x}=x_{1}, \ldots, x_{n}$ an $S$-sequence, and $R=S /(\boldsymbol{x})$ the residue ring. (Namely, let $R$ be a "G-complete intersection.") Then are all bounded totally reflexive $R$-modules eventually periodic (of period 2)?

Let $(S, \mathfrak{n})$ be a regular local ring, $I$ an ideal of $S$ contained in $\mathfrak{n}^{2}$, and $R=S / I$ the residue ring. Then a celebrated theorem of Tate [20] asserts that the ideal $I$ is principal if the residue field of $R$ is bounded as an $R$-module. Combining this with Eisenbud's matrix factorization theorem, we see that $I$ is a principal ideal if and only if every $R$-module in $\mathcal{C}(R)$ is bounded, if and only if every $R$-module in $\mathcal{C}(R)$ is periodic. The question below asks if the G-regular version of this holds. 
Question 6.4. Let $R$ be a local ring over which every totally reflexive module is periodic. Then (under some adequate assumptions) does there exist a G-regular local ring $S$ and an $S$-regular element $f \in S$ such that $R \cong S /(f)$ ? (Namely, is $R$ a "G-hypersurface"?)

A partial answer to this question can be found in [22, Theorem 4.2]. The converse statement holds by Theorem 2.10 and Proposition 2.4. To be precise, let $S$ be a G-regular local ring, $f \in S$ an $S$-regular element, and $R=S /(f)$ the residue ring. Then every totally reflexive $R$-module is periodic.

\section{ACKNOWLEDGMENTS}

The author would like to give his gratitude to Shiro Goto, Yuji Kamoi and Kazuhiko Kurano for valuable discussions and useful suggestions.

\section{REFERENCES}

[1] M. Auslander, Anneaux de Gorenstein, et torsion en algèbre commutative. Séminaire d'Algèbre Commutative dirigé par Pierre Samuel, 1966/67, Texte rédigé, d'après des exposés de Maurice Auslander, Marquerite Mangeney, Christian Peskine et Lucien Szpiro. École Normale Supérieure de Jeunes Filles, Secrétariat mathématique, Paris, 1967.

[2] M. Auslander; M. Bridger, Stable module theory. Memoirs of the American Mathematical Society, No. 94, American Mathematical Society, Providence, R.I., 1969.

[3] L. L. Avramov, Infinite free resolutions. Six lectures on commutative algebra (Bellaterra, 1996), 1-118, Progr. Math., 166, Birkhäuser, Basel, 1998.

[4] L. L. Avramov, Homological dimensions and related invariants of modules over local rings. Representations of algebra. Vol. I, II, 1-39, Beijing Norm. Univ. Press, Beijing, 2002.

[5] L. L. Avramov; A. Martsinkovsky, Absolute, relative, and Tate cohomology of modules of finite Gorenstein dimension. Proc. London Math. Soc. (3) 85 (2002), no. 2, 393-440.

[6] N. Bourbaki, Commutative algebra. Chapters 1-7. Translated from the French. Reprint of the 1989 English translation. Elements of Mathematics (Berlin). Springer-Verlag, Berlin, 1998.

[7] W. Bruns; J. Herzog, Cohen-Macaulay rings. revised edition. Cambridge Studies in Advanced Mathematics, 39, Cambridge University Press, Cambridge, 1998.

[8] L. W. Christensen, Gorenstein dimensions. Lecture Notes in Mathematics, 1747. Springer-Verlag, Berlin, 2000.

[9] L. W. Christensen; G. Piepmeyer; J. Striuli; R. Takahashi, Finite Gorenstein representation type implies simple singularity. Preprint (2007), http://arxiv.org/abs/0704.3421.

[10] D. Eisenbud, Homological algebra on a complete intersection, with an application to group representations. Trans. Amer. Math. Soc. 260 (1980), no. 1, 35-64.

[11] H. KNÖRRER, Cohen-Macaulay modules on hypersurface singularities. I. Invent. Math. 88 (1987), no. 1, 153-164.

[12] H. Matsumura, Commutative algebra. Second edition. Mathematics Lecture Note Series, 56. Benjamin/Cummings Publishing Co., Inc., Reading, Mass., 1980.

[13] R. TAKAhashi, On the category of modules of Gorenstein dimension zero. II. J. Algebra 278 (2004), no. 1, 402-410.

[14] R. Takahashi, Modules of G-dimension zero over local rings of depth two. Illinois J. Math. 48 (2004), no. 3, 945-952.

[15] R. TAKAhAShi, On the category of modules of Gorenstein dimension zero. Math. Z. 251 (2005), no. 2, 249-256.

[16] R. TAKahashi, Remarks on modules approximated by G-projective modules. J. Algebra 301 (2006), no. 2, 748-780.

[17] R. TAKahashi, On the number of indecomposable totally reflexive modules. Bull. London Math. Soc. 39 (2007), no. $3,487-492$.

[18] R. TAKAhashi, An uncountably infinite number of indecomposable totally reflexive modules. Nagoya Math. J. 187 (2007), 35-48.

[19] R. Takahashi; K.-I. Watanabe, Totally reflexive modules constructed from smooth projective curves of genus $g \geq 2$. Arch. Math. (Basel) 89 (2007), no. 1, 60-67.

[20] J. TATE, Homology of Noetherian rings and local rings. Illinois J. Math. 1 (1957), 14-27.

[21] Y. Yoshino, Cohen-Macaulay modules over Cohen-Macaulay rings. London Mathematical Society Lecture Note Series, 146, Cambridge University Press, Cambridge, 1990.

[22] Y. Yoshino, Modules of G-dimension zero over local rings with the cube of maximal ideal being zero. Commutative algebra, singularities and computer algebra (Sinaia, 2002), 255-273, NATO Sci. Ser. II Math. Phys. Chem., 115, Kluwer Acad. Publ., Dordrecht, 2003.

Department of Mathematical Sciences, Faculty of Science, Shinshu University, 3-1-1 Asahi, Matsumoto, NAGANO 390-8621, JAPAN

E-mail address: takahasi@math.shinshu-u.ac.jp 